International Journal of Wireless \& Mobile Networks (IJWMN) Vol. 3, No. 4, August 2011

\title{
PERFORMANCE COMPARISON OF TOPOLOGY AND POSITION BASED ROUTING PROTOCOLS IN VEHICULAR NETWORK ENVIRONMENTS
}

\author{
Akhtar Husain ${ }^{1}$, Ram Shringar Raw ${ }^{2}$, Brajesh Kumar ${ }^{1}$, and Amit Doegar ${ }^{3}$ \\ ${ }^{1}$ Department of Computer Science \& Information Technology, MJP Rohilkhand \\ University, Bareilly, India \\ husainakhtar@yahoo.com, bkumar@mjpru.ac.in \\ ${ }^{2}$ School of Computer and Systems Sciences, Jawaharlal Nehru University, New Delhi, \\ India, \\ rsrao08@yahoo.in \\ ${ }^{3}$ Department of Computer Science, NITTTR, Chandigarh, India \\ amitanitttrchd.ac.in
}

\begin{abstract}
Due to mobility constraints and high dynamics, routing is a more challenging task in VANETs. In this work, we evaluate the performance of the routing protocols in vehicular network environment. The objective of this work is to assess the applicability of these protocols in different vehicular traffic scenarios. Both the position-based and topology-based routing protocols have been considered for the study. The topology-based protocols AODV and DSR and position-based protocol LAR are evaluated in city and highway scenarios. Mobility model has significant effects on the simulation results. We use Intelligent Driver Model (IDM) based tool VanetMobiSim to generate realistic mobility traces. Performance metrics such as packet delivery ratio, throughput, and end-to-end delay are evaluated using NS-2. Simulation results shows position based routing protocols gives better performance than topology based routing protocols.
\end{abstract}

\section{KEYWORDS}

VANET, Routing Protocols, AODV, DSR, LAR, City Scenario, Highway Scenario, Intelligent Driver Model.

\section{INTRODUCTION}

Vehicular ad hoc networks are important technology for future developments of vehicular communication systems. Such networks composed of moving vehicles, are capable of providing communication among nearby vehicles and the roadside infrastructure. Modern vehicles are equipped with computing devices, event data recorders, antennas, and GPS receivers making VANETs realizable. VANETs can be used to support various functionalities such as vehicular safety [1], reduction of traffic congestion, office-on-wheels, and on-road advertisement. Most nodes in a VANET are mobile, but because vehicles are generally constrained to roadways, they have a distinct controlled mobility pattern [2]. Vehicles exchange information with their neighbors and routing protocols are used to propagate information to other vehicles. Some important characteristics that distinguish VANETs from other types of ad hoc networks include:

- $\quad$ High mobility that leads to extremely dynamic topology.

- Regular movement, restricted by both road topologies and traffic rules.

DOI : $10.5121 /$ ijwmn.2011.3420 
- Vehicles have sufficient power, computing and storage capacity.

- Vehicles are usually aware of their position and spatial environment.

Unlike conventional ad hoc wireless networks a VANET not only experiences rapid changes in wireless link connections, but may also have to deal with different types of network topologies. For example, VANETs on freeways are more likely to form highly dense networks during rush hours, while VANETs are expected to experience frequent network fragmentation in sparsely populated rural freeways or during late night. High and restricted node mobility, sort radio range, varying node density makes routing a challenging job in VANETs.

A number of routing protocols have been proposed and evaluated for ad hoc networks. Some of these are also evaluated for VANET environment, but most of them are topology based protocols. Position based protocols (like Location Aided Routing (LAR) [3] [4]), which require information about the physical position of the participating nodes, have not been studied that much and require more attention. The results of performance studies heavily depend on chosen mobility model. The literature shows that the results of many of performance studies are based on mobility models where nodes change their speed and direction randomly. Such models cannot describe vehicular mobility in a realistic way, since they ignore the peculiar aspects of vehicular traffic such as vehicles acceleration and deceleration in the presence of nearby vehicles, queuing at roads intersections, impact of traffic lights, and traffic jams. These models are inaccurate for VANETs and can lead to erroneous results.

In this paper performance evaluation of Ad Hoc On Demand Distance Vector (AODV) [5], Dynamic Source Routing (DSR) [6], and LAR in city and highway traffic environments under different circumstances is presented. To model realistic vehicular motion patterns, we use the Advanced Intelligent Driver Model which is the extension of Intelligent Driver Model (IDM) [7]. We evaluate the performance of AODV, DSR, and LAR in terms of Packet Delivery Ratio (PDR), throughput, and end-to-end delay.

The rest of the paper is organized as follows: Section 2 presents related work, while section 3 briefly depicts the three candidates' protocols. Mobility model for VANET is presented in section 4. Section 5 presents simulation methodology and describes reported results. Finally, in section 6, we draw some conclusion remarks and outline of future works.

\section{RELATED WORK}

Routing protocols can be classified in two broad categories: topology based and position based routing protocols [8] [9]. Topology based approaches, which are further divided into two subcategories: reactive and proactive, use information about links to forward the packets between nodes of the network. Prominent protocols of this category are AODV, and DSR. Position-based routing (e.g. LAR) requires some information about the physical or geographic positions of the participating nodes. In this protocol the routing decision is not based on a routing table but at each node the routing decision is based on the positions of its neighboring nodes and the position of the destination node.

Several studies have been published comparing the performance of routing protocols using different mobility models, and different traffic scenarios with different performance metrics. A paper by Lochert et al. [10] compared AODV, DSR, and Geographic Source Routing (GSR) in city environment. They show that GSR which combines position-based routing with topological knowledge outperforms both AODV and DSR with respect to delivery rates and latency. A study by Jaap et al. [11] examined the performance of AODV and DSR in city traffic scenarios. Another study presented by Juan Angel Ferreiro-Lage et al. [12] compared AODV and DSR 
protocols for vehicular networks and concluded that AODV is best among the three protocols. LAR is described in [3] is to reduce the routing overhead by the use of position information. Position information will be used by LAR for restricting the flooding to a certain area called request zone. Authors found that LAR is more suitable for VANET.

\section{DESCRIPTION OF ROUTING PROTOCOLS}

The topology based routing protocols AODV and DSR are the most studied routing protocols as evident from section 2. The position-based routing protocols need more attention. We have chosen LAR, which is a position-based routing protocol for the performance comparison with AODV and DSR. In this section a brief description of these routing protocols is given.

\subsection{Dynamic Source Routing Protocol (DSR)}

In DSR, when a source node wants to send a packet to a particular destination it checks route cache first. If it does not find any route, it initiates the route discovery protocol. Route discovery involves broadcasting a route request packet. The route request packet contains the address of the source and the destination, and a unique identification number. Each intermediate node checks whether it knows of a route to the destination. If it does not, it appends its address to the route record of the packet and forwards the packet to its neighbors. If the route discovery is successful then the source host, which requested route discovery, receives a route reply packet. Route reply packet lists all the network hops through which target node may be reached. To limit the number of route requests propagated, a node processes the route request packet only if it has not already seen the packet and its own address is not present in the route record of the packet. A route reply is generated when either the destination or an intermediate node with current information about the destination receives the route request packet.

\subsection{Ad Hoc On Demand Distance Vector (AODV)}

In AODV, whenever a source node has to communicate with a destination node such that it has no routing information in its table, it first initiates route discovery process. The node broadcasts a route request (RREQ) packet to all its neighbors. The route request packets contains source address, source sequence number, broadcast ID, destination address, destination sequence number and hop count. The source address and broadcast ID uniquely identifies a RREQ. The source sequence number is for maintaining the freshness of information about the reverse route to the source. The destination sequence number specifies how fresh a route to the destination must be before it can be accepted by the source. If a neighbor knows the route to the destination, it replies with a route reply control message RREP that propagates through the reserve path. Otherwise, the neighbor will re-broadcast the RREQ until an active route is found or the maximum number of hops is reached.

\subsection{Location Aided Routing (LAR)}

The goal of Location-Aided Routing (LAR) described in [3] is to reduce the routing overhead by the use of location information. Position information will be used by LAR for restricting the flooding to a certain area called request zone. As a consequence, the number of route request messages is reduced. Instead of flooding the whole network with route discovery message, this protocol send messages to a subset of nodes from whom the probability of finding route is very high. In LAR, an expected zone is defined as a region that is expected to hold the current location of the destination node. During route discovery procedure, the route request flooding is limited to a request zone, which contains the expected zone and location of the sender node. 
International Journal of Wireless \& Mobile Networks (IJWMN) Vol. 3, No. 4, August 2011

\section{MOBILITY MODEL}

Simulation is a popular approach for evaluating routing protocols; however the accuracy of the results depends on the mobility used. Mobility model has significant effects on the simulation results. Random Waypoint (RWP) model, which is widely used for MANET simulations, is unsuitable for VANET simulations as the mobility patterns underlying an inter-vehicle network are quite different. In order to model realistic vehicular motion patterns The mobility model used for studying VANETs must reflect as close as possible, the behavior of vehicular traffic. Both macro-mobility and micro-mobility aspects need to be considered jointly [13].

\subsection{Macro-Mobility}

Macro-mobility includes all the macroscopic aspects which influence vehicular traffic. The concept of macro-mobility not only takes into account the road topology, but also includes the road structure and characteristics such as unidirectional or bidirectional traffic flows, single or multi-lane, intersection crossing rules, speed limits, vehicle-class based restriction, and the presence of stop signs, traffic lights, etc. The length of streets, the frequency of intersections, and the density of buildings can greatly affect the important mobility metrics such as the speed of the vehicles, or their density over the simulated map.

Path selection strategy is mainly reflected in macro scope. Existing mobility models do not place enough attention on path selection strategies. They simply use shortest path algorithm to compute every node's shortest path. But this approach is not suitable to inter-vehicle communication environment. Vehicles equipped with GPS can get information about local traffic, congestion, and road situation and may choose different path according to their roles even if they have same destination.

\subsection{Micro-Mobility}

The vehicular micro-mobility model includes all aspects related to an individual vehicle's speed and acceleration modeling. When moving along the road, there are typically four state of a vehicle's motion: stop, accelerate, decelerate and move at a constant speed. All vehicles move with changing its motion state, but the changing strategies are different [14]. Acceleration strategy is mainly responsible for speed differences, while stop strategy mainly related to travelling patterns. A vehicle's stop may attribute to reactive reasons or proactive reasons. When a vehicle meets a congestion or accident which blocks it from moving ahead, it is forced to stop. However, a public transport vehicle must stop at every stop for several seconds. These two stop behaviors consume different time.

Micro-mobility refers to drivers' individual behavior, when interacting with other drivers or with the road infrastructure: travelling speed in different conditions; acceleration, deceleration, overtaking criteria, behavior in the presence of road intersections and traffic signs, and general driving attitude. The micro-mobility description plays the main role in the generation of realistic vehicular movements, as it is responsible for effects such as smooth speed variation, vehicles queues, traffic jams and over takings.

\subsection{The Intelligent Driver Model}

The Intelligent Driver Model (IDM) [15] is a car-following model that characterizes drivers' behavior depending on their front vehicles.Vehicles acceleration/deceleration and its expected speed are determined by the distance to the front vehicle and its current speed. Moreover, it is also possible to model the approach of vehicles to crossings. Another advantage of the IDM is that it uses a small set of parameters that which can be evaluated with the help of real traffic 
measurements. The instantaneous acceleration of a vehicle is computed according to the following equation:

$$
\frac{d v}{d t}=a\left[1-\left(\frac{v}{v_{0}}\right)-\left(\frac{S^{*}}{S}\right)^{2}\right]
$$

Where $v$ is the current speed of the vehicle, $v_{0}$ is the desired velocity, $S$ is the distance from the preceding vehicle and $S^{*}$ is the desired dynamical distance to the vehicle/obstacle, which computed with the help of equation (2).

$$
S^{*}=S_{0}+\left[v T+\left(\frac{v \cdot \Delta v}{2 \sqrt{ } a b}\right)\right]
$$

Desired dynamical distance $S^{*}$ is a function of jam distance $S_{0}$ between two successive vehicles, the minimum safe time headway $T$, the speed difference with respect to front vehicle velocity $\Delta v$, maximum acceleration $a$, and maximum deceleration $b$.

VanetMobiSim developed by J. Harri et al [16] [17], extends IDM and adds two new microscopic mobility models Intelligent Driver Model with Intersection Management (IDM-IM) and Intelligent Driver Model with Lane Changing (IDM-LC). IDM-IM adds intersection handling capabilities to the behavior of vehicles driven by the IDM. Moreover, IDM-IM models two different intersection scenarios: a crossroad regulated by the stop signs, and a road junction ruled by traffic lights. IDM-LC extends the IDM-IM model with the possibility for the vehicles to change lane and overtake each others.

\section{EXPERIMENTS AND EVALUATIONS}

Extensive simulations have been carried out to evaluate and compare the performances of LAR, AODV, and DSR in VANETs by using the network simulator NS-2 in its version 2.32. It is freely available and widely used for research in mobile ad hoc networks. The movements of nodes are generated using VanetMobiSim tool [18]. The awk programming is used to analyze the simulation results. It is assumed that every vehicle is equipped with GPS and can obtain its current location.

\subsection{System Model}

Vehicles are deployed in a $1000 \mathrm{~m} * 1000 \mathrm{~m}$ area. A Manhattan grid like road network is assumed to have eight vertically and horizontally oriented roads and 16 crossings. The vehicle moves and accelerates to reach a desired velocity. When a vehicle moves near other vehicles, it tries to overtake them if road includes multiple lanes. If it cannot overtake it decelerate to avoid the impact.

Table 1. Mobility model parameters

\begin{tabular}{|l|l|}
\hline Parameter & Value \\
\hline Maximum Acceleration & $0.9 \mathrm{~m} / \mathrm{s}^{2}$ \\
\hline Maximum Deceleration & $0.5 \mathrm{~m} / \mathrm{s}^{2}$ \\
\hline Maximum safe deceleration & $4 \mathrm{~m} / \mathrm{s}^{2}$ \\
\hline Vehicle Length & $5 \mathrm{~m}$ \\
\hline Traffic light transition & $10 \mathrm{~s}$ \\
\hline
\end{tabular}


International Journal of Wireless \& Mobile Networks (IJWMN) Vol. 3, No. 4, August 2011

\begin{tabular}{|l|l|}
\hline Lane change threshold & $0.2 \mathrm{~m} / \mathrm{s}^{2}$ \\
\hline Politeness & 0.5 \\
\hline Safe headway time & $1.5 \mathrm{~s}$ \\
\hline Maximum congestion distance & $2 \mathrm{~m}$ \\
\hline
\end{tabular}

When a vehicle is approaching an intersection, it first acquires the state of the traffic sign. If it is a stop sign or if the light is red, it decelerates and stops. If it is a green traffic light, it slightly reduces its speed and proceeds to the intersection. The other mobility parameters are given in table 1.

Table 2. Simulation parameters

\begin{tabular}{|l|l|}
\hline Parameter & Value \\
\hline MAC and Channel Type & IEEE 802.11 ,Wireless Channel \\
\hline Antenna type & Omni directional \\
\hline Simulation time & 1500 seconds \\
\hline Simulation area & $1000 \mathrm{~m}$ x $1000 \mathrm{~m}$ \\
\hline Transmission range & $250 \mathrm{~m}$ \\
\hline Node speed & $30 \mathrm{~km} / \mathrm{hr}$ and $100 \mathrm{~km} / \mathrm{hr}$ \\
\hline Traffic type & CBR \\
\hline Data payload & 512 bytes/packet \\
\hline Packet rate & 4 packets/sec \\
\hline Node pause time & $20 \mathrm{~s}$ \\
\hline Bandwidth & 2 Mbps \\
\hline Mobility model & IDM based \\
\hline Interface queue type & Drop Tail/Priority Queue, and CMU Priori Queue \\
\hline Interface queue length & 50 packets \\
\hline Receiving threshold & Two-Ray Rx Power (Radio Range) \\
\hline Sensing threshold & Two-Ray Rx Power (Radio Range + 5 m) \\
\hline No. of vehicles & 10 to 80 \\
\hline
\end{tabular}

Vehicles are able to communicate with each other using the IEEE 802.11 DCF MAC layer. The transmission range is taken to be 250 meters. The traffic light period is kept constant at 60 seconds. Simulations are repeated varying the speed, that is, $30 \mathrm{~km} / \mathrm{h}$ (city) and $100 \mathrm{~km} / \mathrm{h}$ (highway) and varying the node density. The other simulation parameters are given in Table 2. Only one lane case is taken for city scenario. However, in highway scenario, first one lane case is considered and later it is generalized to multiple lanes. The other simulation parameters are given in Table 2.

\subsection{Results and Discussion}

The protocols are evaluated for packet delivery ratio, throughput, and average end-to-end delay at varying node densities (10 to 80 vehicles). Hereafter the terms node and vehicle are used interchangeably.

\subsubsection{Packet Delivery Ratio (PDR)}

Packet delivery ratio is defined as the ratio of data packets received by the destinations to those generated by the sources. Mathematically, it can be defined as: 
International Journal of Wireless \& Mobile Networks (IJWMN) Vol. 3, No. 4, August 2011

$$
P D R=\frac{S_{a}}{S_{b}}
$$

Where, $S_{a}$ is the sum of data packets received by the each destination and $S_{b}$ is the sum of data packets generated by the each source.

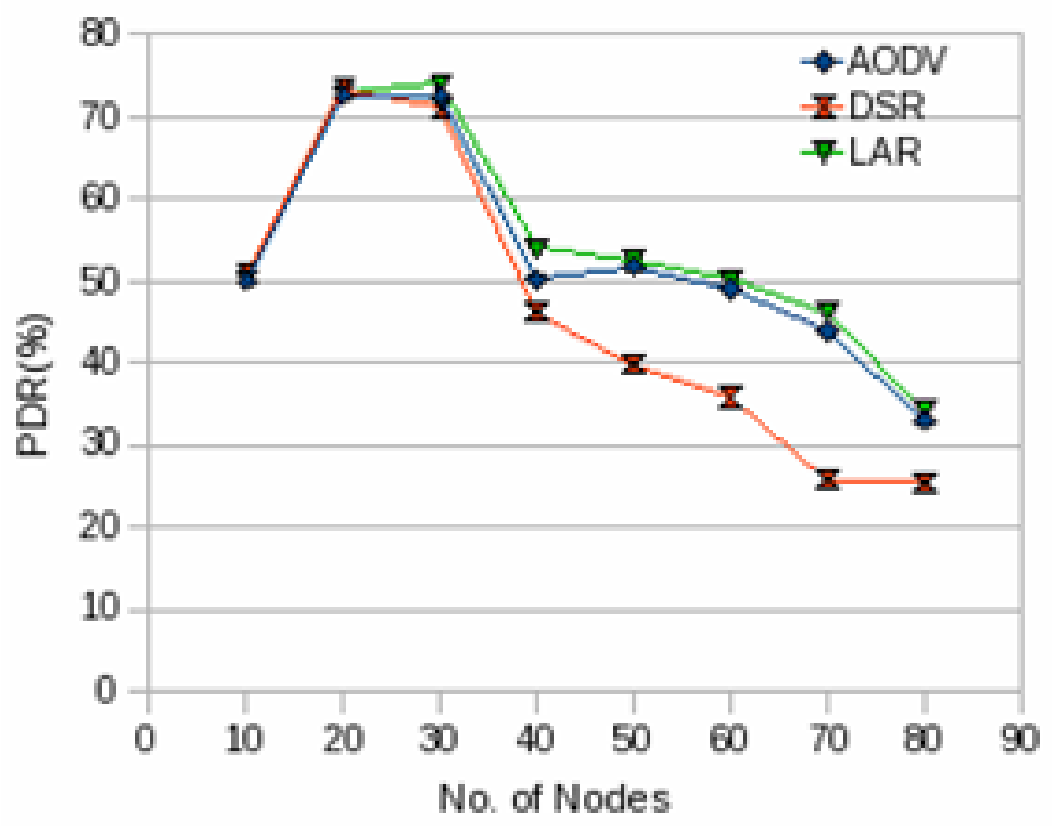

(a) City Scenario using IDM-IM



(b) Highway Scenario using IDM-IM 
International Journal of Wireless \& Mobile Networks (IJWMN) Vol. 3, No. 4, August 2011

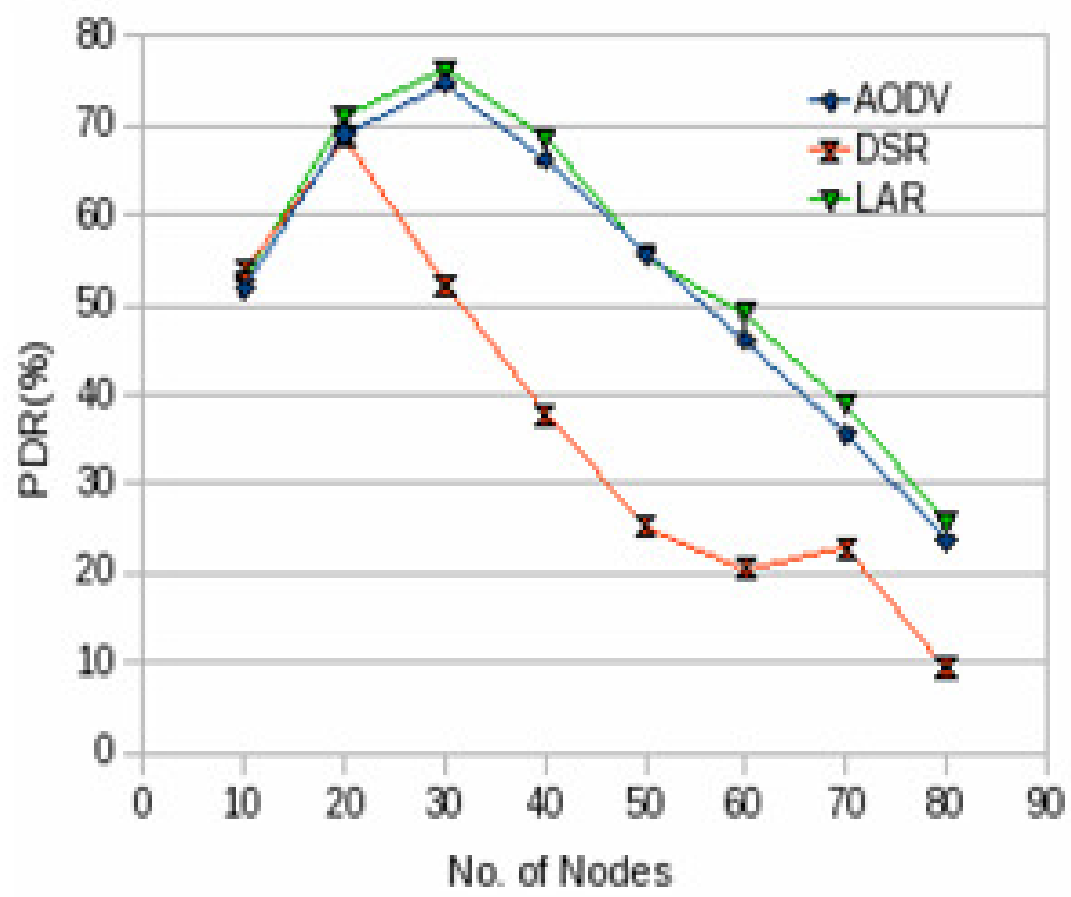

(c) Highway Scenario using IDM-LC (lane = 2)

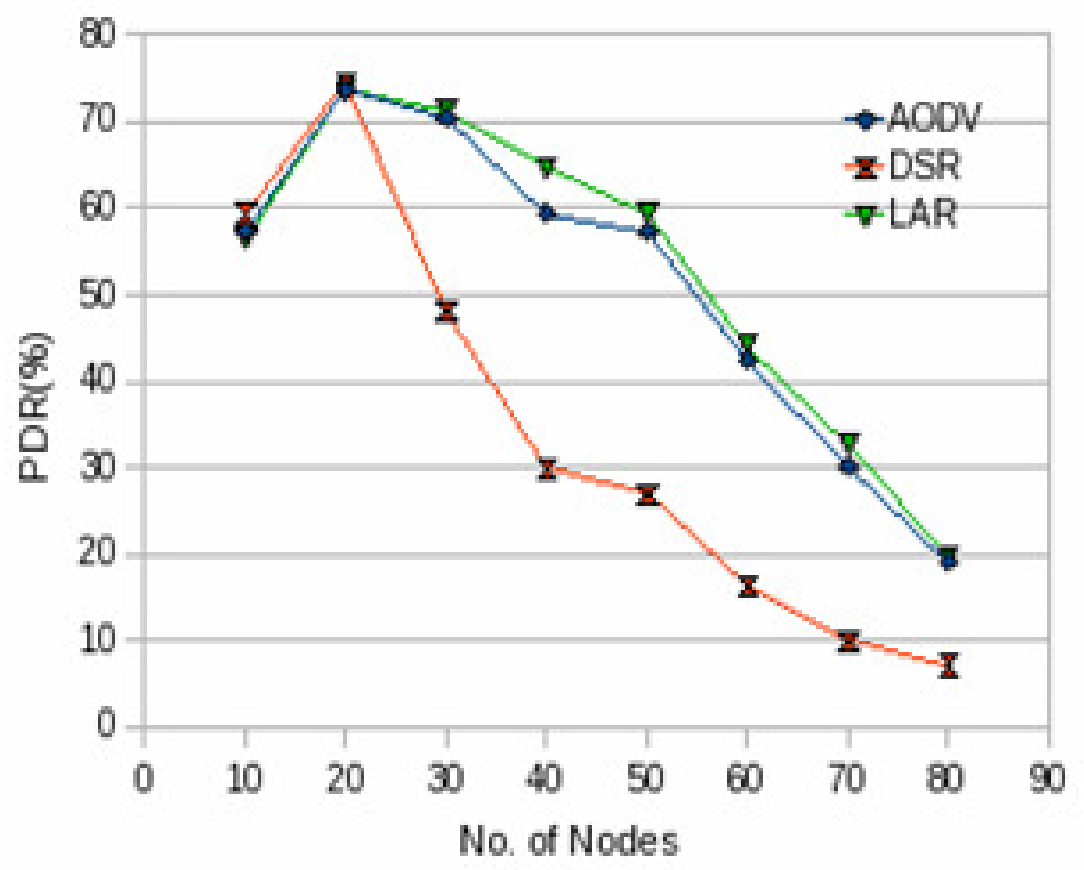

(d) Highway Scenario using IDM-LC (lane = 4)

Figure 1. PDR as a function of node density 
Figure 1 depicts the fraction of data packets that are successfully delivered during simulations time versus the number of nodes. In city scenario all the four protocols exhibit good performance for low node densities as shown in Figure 1(a) and none of the protocols clearly outperforms the others. But with the increase in density, performance of the protocols decreases. It can be observed that the performance of the DSR reduces drastically while LAR is slightly better among the three. In highway scenario all the protocols show relatively better performance as depicted by Figure 1(b) and location based protocol clearly outperform the topology-based protocols with LAR exhibiting best results. Though, PDR again decreases with increasing density, but it is not that much low as observed in city scenario. Figure 1(c) and Figure 1(d) show that performance of the protocols degrades in highway scenario with lane changing (LC). It further slightly degrades with increase in the number of lanes. Again DSR is the worst and LAR has a slight edge over the other protocols.

\subsubsection{Throughput}

Number of bits delivered successfully per second to the destination. It is the sum of bits received successfully by all destinations. It is represented in kilo bits per second (kbps). Mathematically, it can be defined as:

$$
\text { Throughput }=\frac{N}{1000}
$$

Where $N$ is the number of bits received successfully by all destinations.

Figure 2 illustrates the variation of throughput by varying node densities. Results show that throughput of all the candidate protocols clearly increases between densities of 10 and 30 nodes. This behavior results from the network connectivity. When density is low, in many cases it may not be possible to establish a connection between source and destination pair.

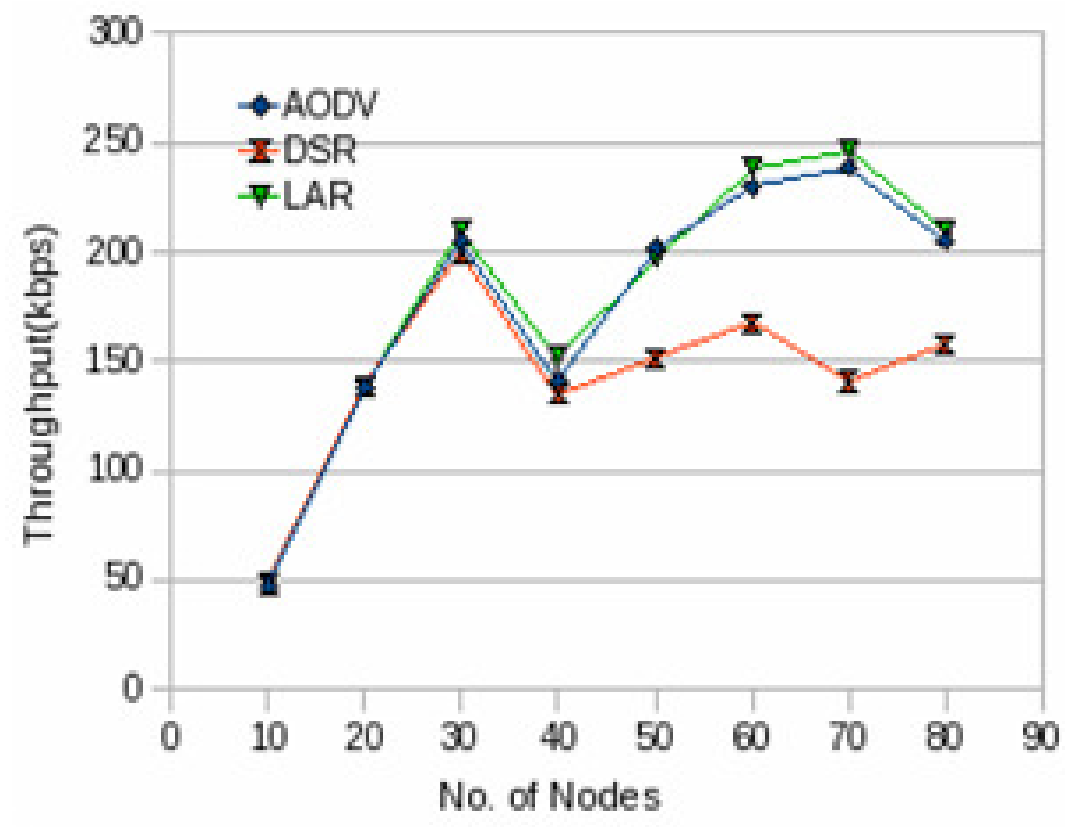

(a) City scenario using IDM-IM 
International Journal of Wireless \& Mobile Networks (IJWMN) Vol. 3, No. 4, August 2011

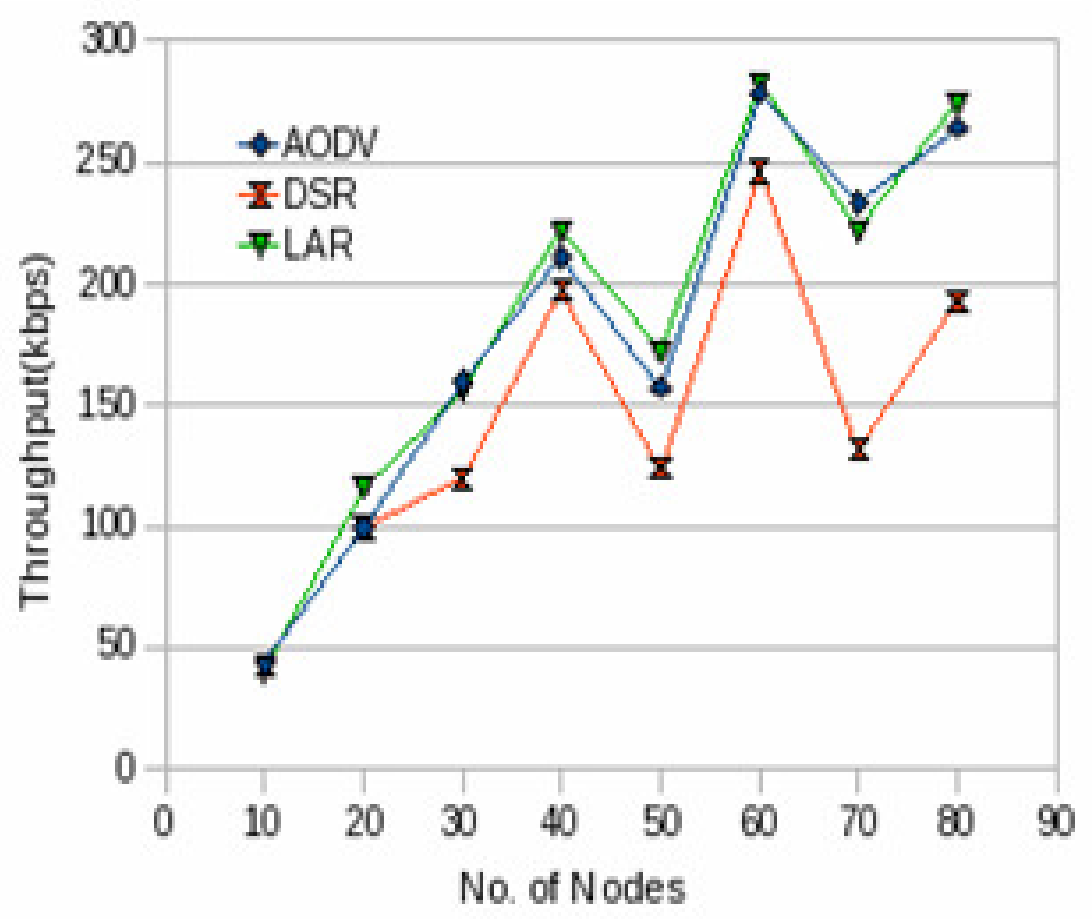

(b) Highway scenario using IDM-IM



(c) Highway scenario using IDM-LC (lane = 2) 


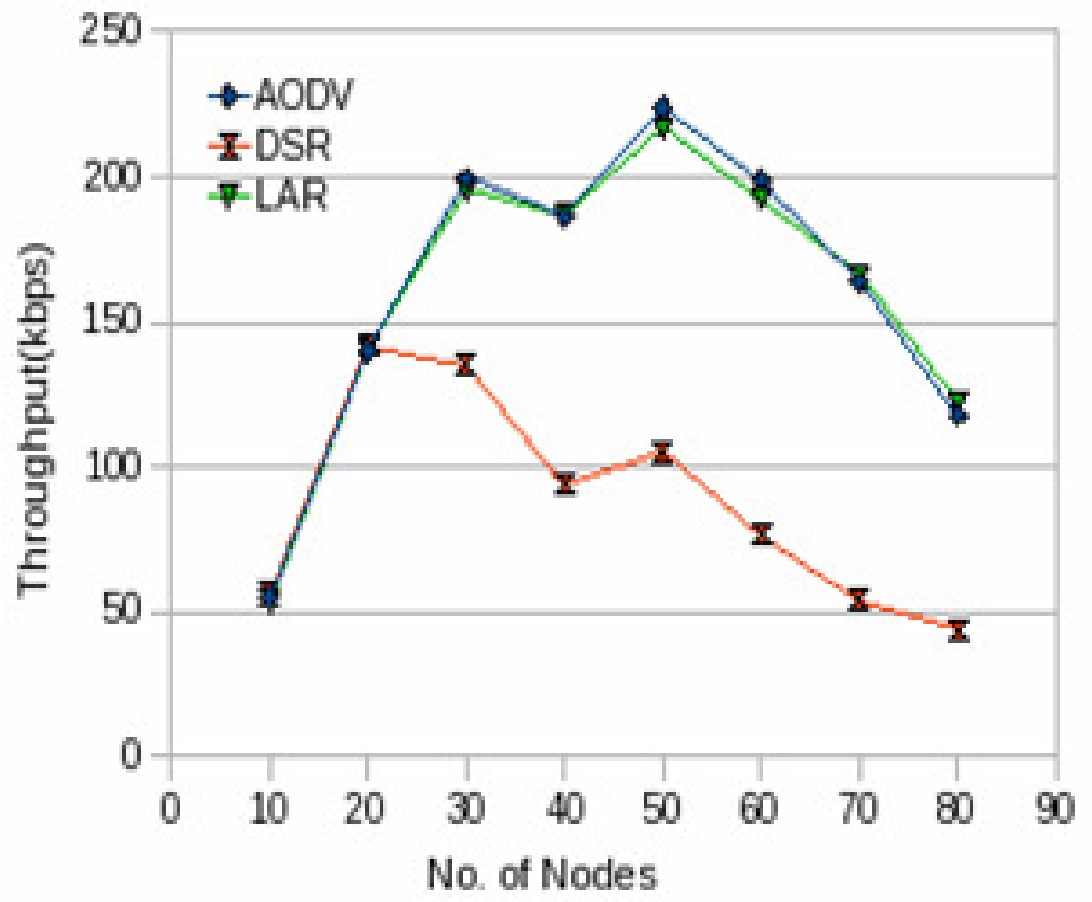

(d) Highway scenario using IDM-LC (lane =4)

Figure 2. Throughput vs node densities

Throughput improves when connectivity is better. At higher densities, the throughput of all the protocols is reduced. It is due to increased routing overhead when wireless channel is shared by more and more vehicles. Again DSR is not coming good and LAR clearly outperforms the other protocols, especially beyond density of 30 nodes.

\subsubsection{Average End-to-End Delay (EED)}

The average time it takes a data packet to reach the destination. This includes all possible delays caused by buffering during route discovery latency, queuing at the interface queue, retransmission delay at MAC, and propagation delay. This metric is calculated by subtracting time at which first packet was transmitted by source from time at which first data packet arrived to destination. Mathematically, it can be defined as:

$$
\text { Average EED }=\frac{S}{N}
$$

Where $S$ is the sum of the time spent to deliver packets for each destination, and $N$ is the number of packets received by the all destination nodes.

Finally, Figure 3 summarizes the variation of the average latency by varying node density. Average latency increases with increasing the number of nodes. DSR consistently presents the highest delay. This may be explained by the fact that its route discovery process takes a quite long time compared to other protocols. LAR has the lowest delay though compared to DSR and AODV it is low only slightly. In city scenario the location-based protocol exhibit lower delay 
International Journal of Wireless \& Mobile Networks (IJWMN) Vol. 3, No. 4, August 2011

compared to AODV especially at higher densities. Delay of all the protocols clearly increases in highway scenario with lane changing.

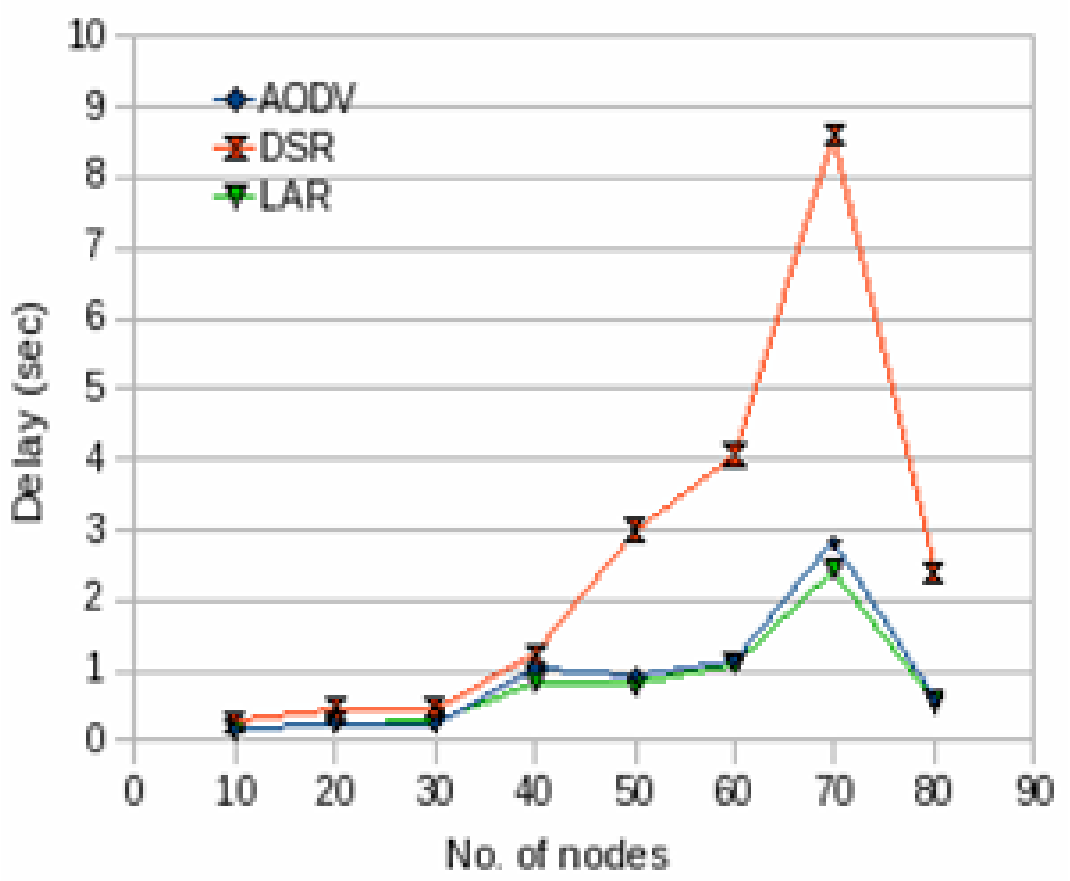

(a) City scenario using IDM-IM

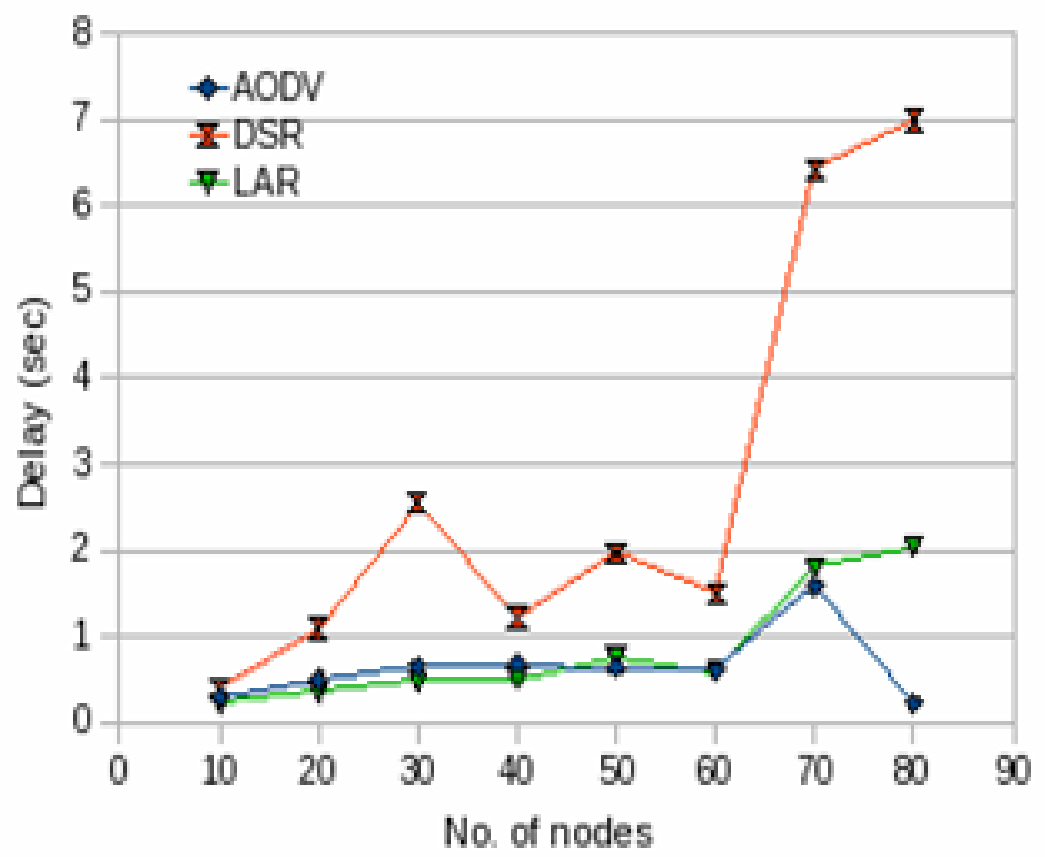

(b) Highway scenario using IDM-IM 
International Journal of Wireless \& Mobile Networks (IJWMN) Vol. 3, No. 4, August 2011

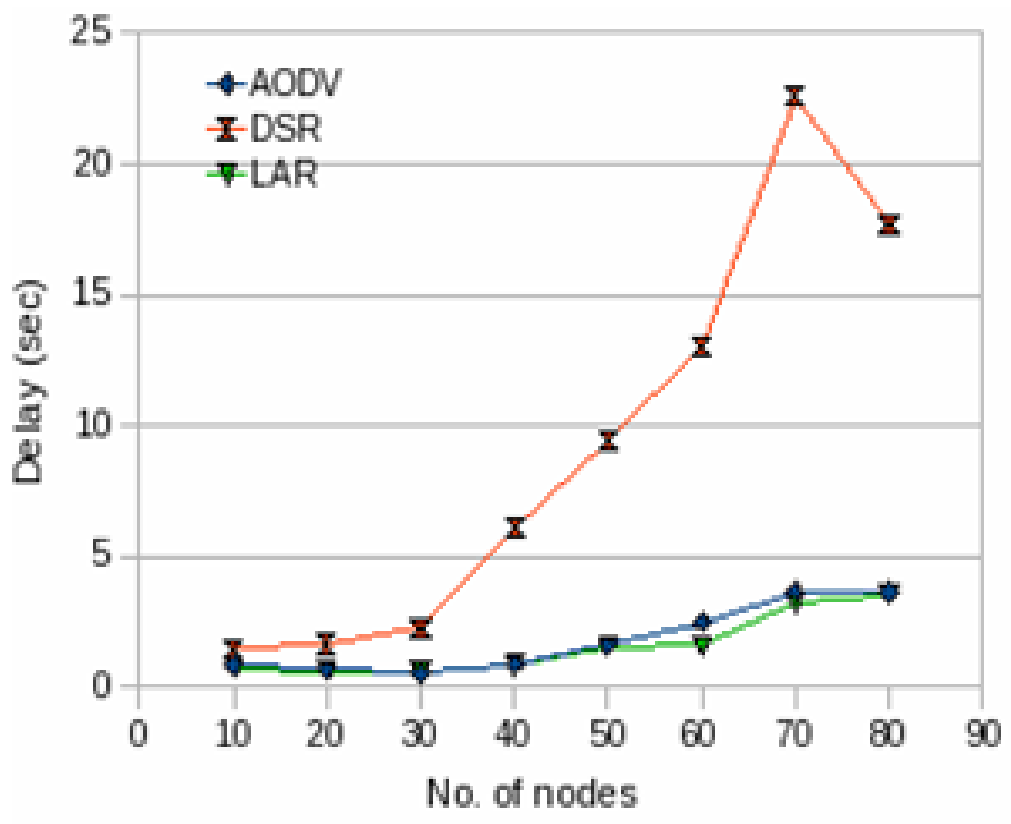

c) Highway scenario using IDM-LC (lane $=2)$

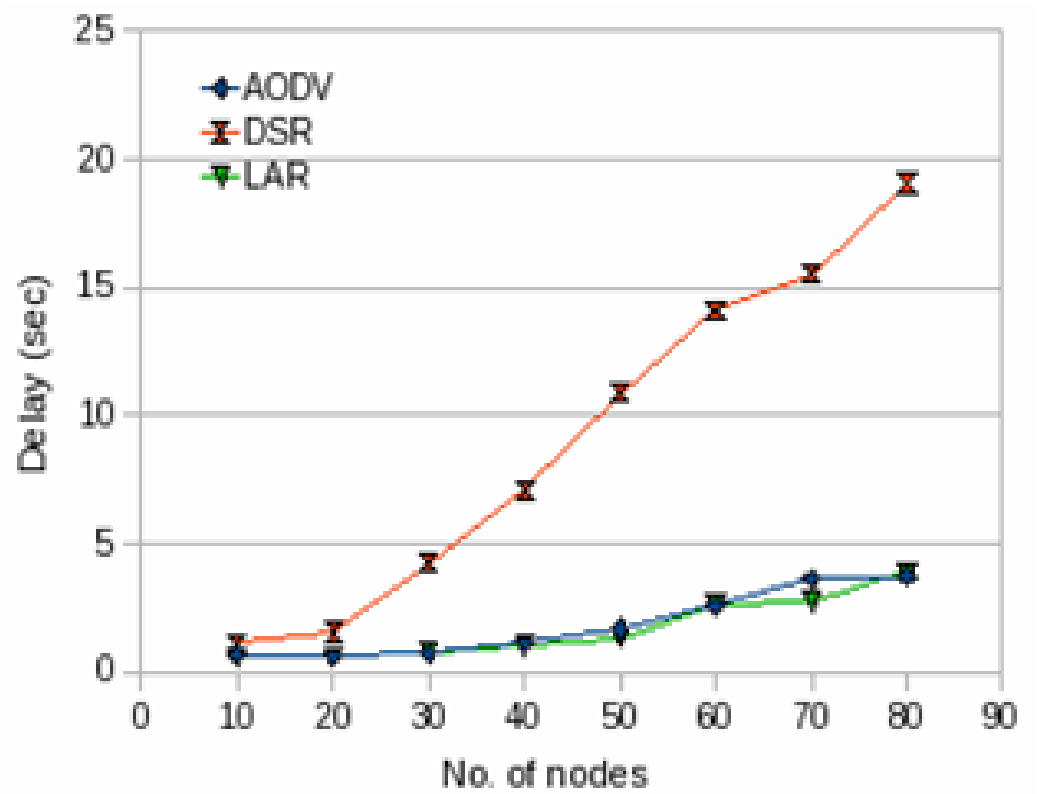

(d) Highway scenario using IDM-LC (lane $=4)$

Figure 3. Delay as a function of node density

Overall, it can be concluded that LAR showed the best performance in the simulated scenarios with highest PDR. Moreover, it has the highest throughput and shown the lower delays also. It can be said that DSR is not a suitable protocol for VANET whether it is city scenario or highway scenario. AODV also showed the good performance with better PDR and throughput and lower delays. 


\section{CONCLUSION AND FUTURE WORK}

In this paper, performance of three routing protocols AODV, DSR, and LAR were evaluated for vehicular ad hoc networks in city and highway scenarios. We used Advanced Intelligent Driver Model to generate realistic mobility patterns. The three protocols were tested against node density for various metrics. It is found that position based routing protocol (LAR) outperforms topology based routing protocols (DSR and AODV) in different VANET environment. For most of the metrics LAR has the better performance.

Overall, it can be concluded that position based routing protocol gives better performance than topology based routing protocols in terms of packet delivery ratio, throughput, and end-to-end delay for both the vehicular traffic scenarios. For future work, we want to study the impact of traffic signs, traffic lights and transmission range on the performance of the routing protocols. Future work will also include the evaluation of other position based routing protocols as they are more suitable in vehicular traffic environment.

\section{REFERENCES}

[1] X. Yang, J. Liu, F. Zhao, and N. Vaidya, (2004) “A Vehicle-to Vehicle Communication Protocol for Cooperative Collision Warning”, Int'l Conf. On Mobile and Ubiquitous Systems: Networking and Services (MobiQuitous 2004), pp. 44-45.

[2] J. Bernsen, and D. Manivannan, (2009) "Unicast routing protocols for vehicular ad hoc networks", A critical comparison and classification, Elsevier Journal of Pervasive and Mobile Computing,vol. 5, issue 1, pp. 1-18.

[3] Y. Ko, and N. Vaidya, (2002) "Location Aided Routing in Mobile Ad Hoc Networks", ACM journal of Wireless Networks, vol. 6, no. 4, pp 307-321.

[4] T. Camp, J. Boleng, B. Williams, L. Wilcox, and W. Navidi, (2002) "Performance Comparison of Two Locations Based Routing Protocols for Ad Hoc Networks", Proceedings of the IEEE INFOCOM,vol.3, pp. 1678-1687.

[5] C. E. Perkins, and E. M. Royer, (1999) "Ad hoc on-demand distance vector routing", Proceedings of the 2 IEEE Workshop on Mobile Computing Systems and Applications, New Orleans, I.A. pp. 90-100.

[6] D. B. Johnson, D. A. Maltz, and J. Broch, (1985) "Dynamic source routing protocol for wireless ad hoc network", IEEE Journal on Selected Areas in Communication, Vol. 3, Issue 3, pp.431439.

[7] J. Haerri, F. Filali, and C. Bonnet, (2006) "Performance comparison of AODV and OLSR in VANETs urban environments under realistic mobility patterns", 5 annual IFIP Mediterranean Ad Hoc Networking Workshop (Med-Hoc-Net 2006), Lipari, Italy.

[8] M. Mauve, J. Widmer, and H. Hartenstein, (2001) "A survey on position-based routing in mobile ad hoc networks", IEEE Network Magazine, Vol. 15, Issue No.6, pp. 30-39.

[9] X. Hong, K. Xu, and M. Gerla, (2002) "Scalable Routing Protocols for Mobile Ad Hoc Networks”, IEEE Network Magzine, vol. 16, no. 4, pp 11-21.

[10] C. Lochert, H. Hartenstein, and J. Tian, (2003) "A Routing strategy for vehicular ad hoc networks in city environments", Proceedings of IEEE Intelligent Vehicles Symposium, Columbus, USA, pp. 156-161.

[11] S. Jaap, M. Bechler, and L. Wolf, (2005) "Evaluation of routing protocols for vehicular ad hoc networks in city traffic scenarios", Proceedings of the 5th International Conference on Intelligent Transportation Systems Telecommunications (ITST),Brest, France. 
International Journal of Wireless \& Mobile Networks (IJWMN) Vol. 3, No. 4, August 2011

[12] J. A. Ferreiro-Lage, C. P. Cristina Pereiro Gestoso, O. Rubinos, and F. A. Agelet, (2009) "Analysis of Unicast Routing Protocols for VANETs", 5th Intl. Conference on Networking and Services, Valencia Spain, pp 518-521.

[13] S. Xi, and X. Li, (2008) "Study of the Feasibility of VANET and its Routing Protocols", Proceedings of 4th Int. Conference, WiCOM'08, Dalian, pp 1-4.

[14] M. Bakhouya, J. Gaber, and M. Wack, (2009) "Performance evaluation of DREAM protocol for Inter-Vehicle Communication", 1 Intl. Conference on Wireless Communications, Vehicular Tech, Information Theory, and Aerospace \& Electronics Systems Technology, Wireless VITAE 2009,Aalborg, pp 289-293.

[15] M. Trieber, A. Hennecke, and D. Helbing, (2000) "Congested traffic states in empirical observations and microscopic simulations”, Phys. Rev. E 62, Issue 2.

[16] J. Harri, and M. Fiore, (2006) "VanetMobiSim- vehicular ad hoc network mobility extension to the CanuMobiSim framework", Manual, Institute Eurecom/Politecnico di Torino, Italy.

[17] The Network Simulator NS-2 (2006), URL: http://www.isi.edu/nsnam/ns/.

[18] VanetMobiSim, URL: http://vanet.eurecom.fr/.

Authors:

Akhtar Husain did B.E. in Computer Science \& Engineering from GBPEC Pauri Garhwal (H.N.B. Garhwal Central University), India in 1996 and M.E. in Computer Science \& Engineering from National Institute of Technical Teacher's Training and Research (Panjab University) Chandigarh, India. He is also working as Assistant Professor in the Department of Computer Science \& Information Technology at MJP Rohilkhand University, Bareilly, India. His research interests include mobile ad hoc networks, network security, fuzzy logic and neural networks.

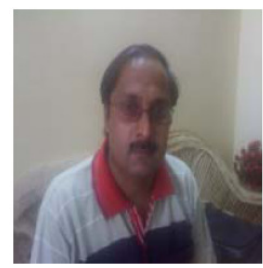

Ram Shringar Raw received his B. E. (Computer Science and Engineering) from G. B. Pant Engineering College, Pauri-Garhwal, UK, India and M. Tech (Information Technology) from Sam Higginbottom Institute of Agriculture, Technology and Sciences, Allahabad (UP), India in 2000 and 2005, respectively. He has submitted his Ph.D (Computer Science and Technology) to School of Computer and Systems Sciences, Jawaharlal Nehru University, New Delhi, India. He is currently working as Assistant Professor at Department of Computer Science and Engineering, Ambedkar Institute of Technology, GGSIP University, New Delhi,

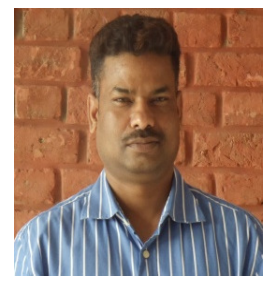
India. His current research interest includes Mobile Ad hoc Networks and Vehicular Ad hoc Networks. Mr. Raw has published papers in International Journals and Conferences including IEEE, Springer, Inderscience, AIRCC, etc.

Brajesh Kumar received his M. Tech in Computer Science \& Technology from Indian Institute of Technology, Roorkee, India in 2001. He is with the Department of Computer Science \& Information Technology, MJP Rohilkhand University, Bareilly, India since 2002. His research interests include mobile ad hoc networks, sensor networks, and network security.

Amit Doegar received his M.E. in Computer Science \& Engineering from National Institute of Technical Teacher's Training and Research (Panjab University) Chandigarh, India in 2008. He is working as an Assistant Professor in the Department of Computer Science, NITTTR Chandigarh, India. His research interests include Internet, web technologies, wireless and mobile networks.
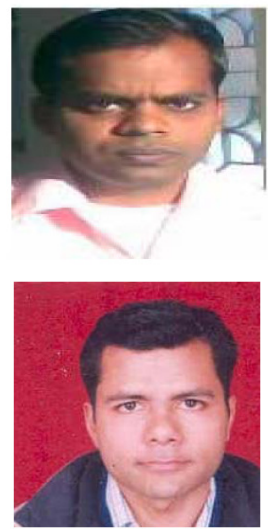